\title{
Status and Results from the EXO Collaboration
}

\author{
Joshua Albert ${ }^{1, a}$ \\ ${ }^{1}$ Department of Physics, Indiana University, Bloomington, IN, USA
}

\begin{abstract}
The Enriched Xenon Observatory (EXO) is an experimental program searching for neutrinoless double-beta decay using ${ }^{136} \mathrm{Xe}$. Such a search can shed light on the Majorana nature of the neutrino (whether the neutrino is its own anti-particle), the absolute mass scale of neutrinos, and beyond standard model processes that violate lepton number conservation. The first phase of the experiment, EXO-200, uses $200 \mathrm{~kg}$ of xenon with $80 \%$ enrichment in ${ }^{136} \mathrm{Xe}$ in a single-phase liquid xenon time projection chamber (TPC). The double-beta decay of xenon is detected in the ultra-low background TPC by collecting both the scintillation light and the ionization charge. The detector has been taking low background physics data with enriched xenon at the Waste Isolation Pilot Plant (WIPP) in New Mexico since early May 2011. The results produced from the collaboration include the first observation of two-neutrino double-beta decay of ${ }^{136} \mathrm{Xe}$, and a neutrinoless double-beta decay search result that places one of the most stringent limits on the effective Majorana neutrino mass. Building on the success of EXO-200, the collaboration is performing feasibility studies and $R \& D$ work for a future multi-tonne scale experiment named nEXO. During the talk, I will discuss the latest results from EXO-200 and prospects of neutrinoless double-beta decay search with both EXO-200 and nEXO.
\end{abstract}

\section{Introduction}

Neutrinos are some of the most difficult known particles to study. Their mass is presently unknown (although we know they do have mass from oscillation experiments), as is their potential to undergo CP-violating transformations. Their extremely low mass is one of the challenges to the standard model. Possibly even more fundamentally, we do not know whether neutrinos are Dirac or Majorana particles. Dirac particles (such as electrons and quarks) are distinct from their anti-particles (positrons and anti-quarks). Majorana particles are not distinct from their anti-particles. Photons and pi-zero particles are like this, though neither is a fundamental fermion. It is currently unknown whether any Majorana fermions exist. Neutrinos, with their lack of electric charge, could be Majorana in nature, in which case the only real difference between a neutrino and an anti-neutrino (as we have observed them) would be the particle spin.

The most promising avenue to get an answer to this question is through a search for neutrinoless double-beta decay $(0 v \beta \beta)$. In certain nuclei with an even number of both protons and neutrons, ordinary beta decay may be energetically forbidden,

$$
n \rightarrow p+e^{-}+\bar{v}_{e}
$$

\footnotetext{
a e-mail: joalbert@indiana.edu
} 
but double-beta decay $(2 v \beta \beta)$ may be allowed,

$$
2 n \rightarrow 2 p+2 e^{-}+2 \bar{v}_{e}
$$

This second-order weak interaction is rare, but has been observed for some isotopes, including ${ }^{136} \mathrm{Xe}$.

If neutrinos are Majorana particles, it should be possible for one of the two anti-neutrinos in the $2 v \beta \beta$ decay to act as a neutrino, and cancel out the other anti-neutrino. This interaction (Figure 1) is neutrinoless double-beta decay. The signature to observe this interaction is that the two emitted electrons will have the full Q-value energy, as none of it is lost to the anti-neutrinos, as it is in $2 v \beta \beta$.

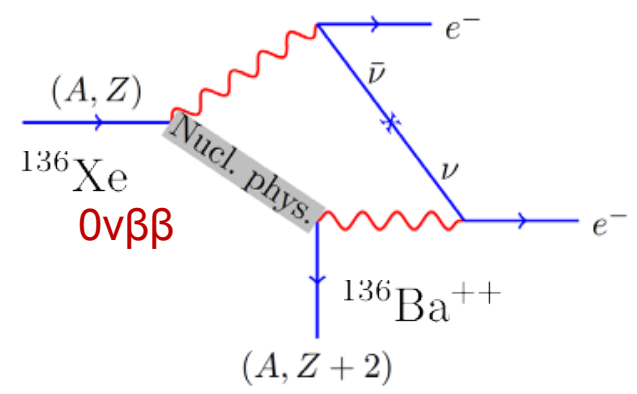

Figure 1. Diagram of a simple mechanism for neutrinoless double-beta decay. Note that the neutrino can only be produced if it is equivalent to the anti-neutrino, that is, a Majorana fermion.

If observed, $0 v \beta \beta$ would be a lepton-number violating process, and require physics beyond the standard model. Observation would clearly indicate that neutrinos are Majorana particles, and, if the mass of the neutrino were known and sufficiently large, non-observation of $0 v \beta \beta$ would clearly indicate that neutrinos are Dirac particles. The lifetime for $0 v \beta \beta$ is related to the neutrino mass by

$$
\frac{1}{t_{1 / 2}^{0 v}}=G^{0 v}\left|M^{0 v}\right|^{2} m_{\beta \beta}^{2},
$$

where $G^{0 v}$ is a phase space factor, $M^{0 v}$ is a nuclear matrix element, and $m_{\beta \beta}$ is given by

$$
m_{\beta \beta}=\left|\sum_{i=1}^{3} m_{i} U_{e i}^{2}\right|
$$

and is the effective mass of the electron neutrino. Thus, observation of $0 v \beta \beta$ and measurement of its rate would also be a measurement of the neutrino mass.

\section{EXO-200 Detector}

The Enriched Xenon Observatory (EXO) collaboration is searching for $0 v \beta \beta$ in ${ }^{136} \mathrm{Xe}$. This isotope was chosen for several reasons:

- It is relatively easy to enrich, as it is the heaviest long-lived isotope of xenon.

- It can be both the source and detection medium (based on scintillation light and ionization of liquid xenon), yielding a monolithic detector. 
- It can be continuously circulated and purified.

- It has a reasonably high Q-value $(2457.8 \mathrm{keV})$, well above the energies of most background gammas.

- It may be possible to tag the resulting ${ }^{136} \mathrm{Ba}^{++}$daughter.

The EXO-200 detector is a liquid xenon time projection chamber (TPC), enriched to $80.6 \%{ }^{136} \mathrm{Xe}$. A voltage gradient of $376 \mathrm{~V} / \mathrm{cm}$ between the central cathode and the anode planes near the endcaps serve to drift charge from ionization to the induction and collection wire-planes. Avalanche photodiodes (APDs) on the endcaps of the cylindrical TPC collect scintillation light. The combined information of the crossed induction and charge collection wire-planes, along with the scintillation light, and the timing difference between scintillation and charge collection, allows 3-D reconstruction of events in the TPC. More information on the EXO-200 TPC can be found in [1]. A photograph of the inside of the TPC (during assembly) is shown in Figure 2.

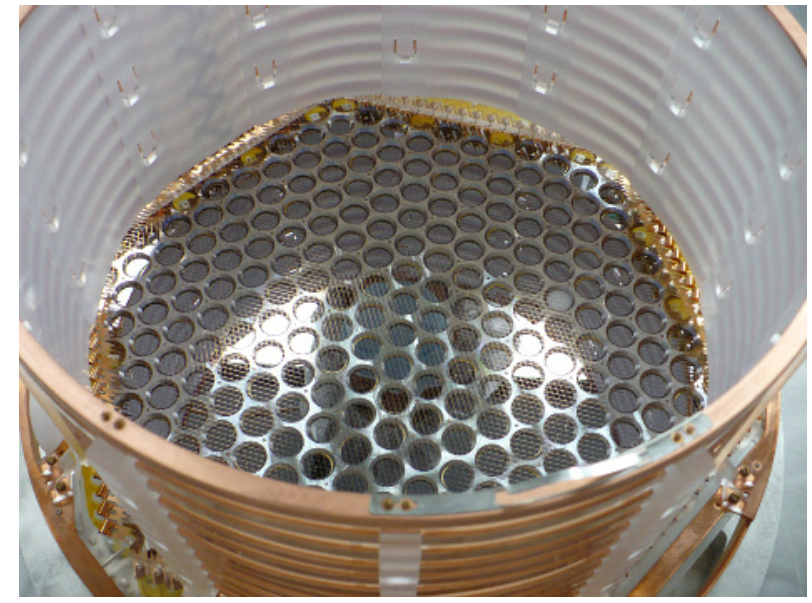

Figure 2. Photograph of the inside of the EXO-200 TPC, taken during assembly. This is one half of the TPC. The crossed induction wire plane and collection wire plane are visible above the APD plane. The copper field rings surround the cylindrical TPC, with white teflon reflector just inside of their radius.

The EXO-200 detector is installed underground (1600 meters water equivalent depth) at the Waste Isolation Pilot Plant (WIPP), near Carlsbad, NM. The TPC is enclosed in a cryostat (for temperature regulation as well as shielding), which is surrounded by further lead shielding. A drawing of the detector and shielding setup is in Figure 3.

A big feature of the TPC is that the scintillation and ionization channels can be combined to get an improved energy resolution [2]. This effect is demonstrated in the thorium source calibration data, shown in Figure 4. The calibration sources are fed into the cryostat through a copper source tube that passes through the shielding and enters the cryostat, winding around the TPC. The gamma lines from ${ }^{228} \mathrm{Th},{ }^{137} \mathrm{Cs}$, and ${ }^{60} \mathrm{Co}$ sources are used to map out detector response.

Another useful feature of the TPC is that gamma backgrounds can largely be rejected by a multiplicity cut. Gammas tend to Compton scatter and deposit charge at multiple positions in the TPC (known as multi-site, MS, events). Sometimes the clusters are too close together to resolve, resulting in a single-site (SS) event, but we can reduce gamma backgrounds at the Q-value by near a factor of 5 by rejecting MS events. Double-beta decays at the Q-value are expected to be $82.5 \% \mathrm{SS}$. 


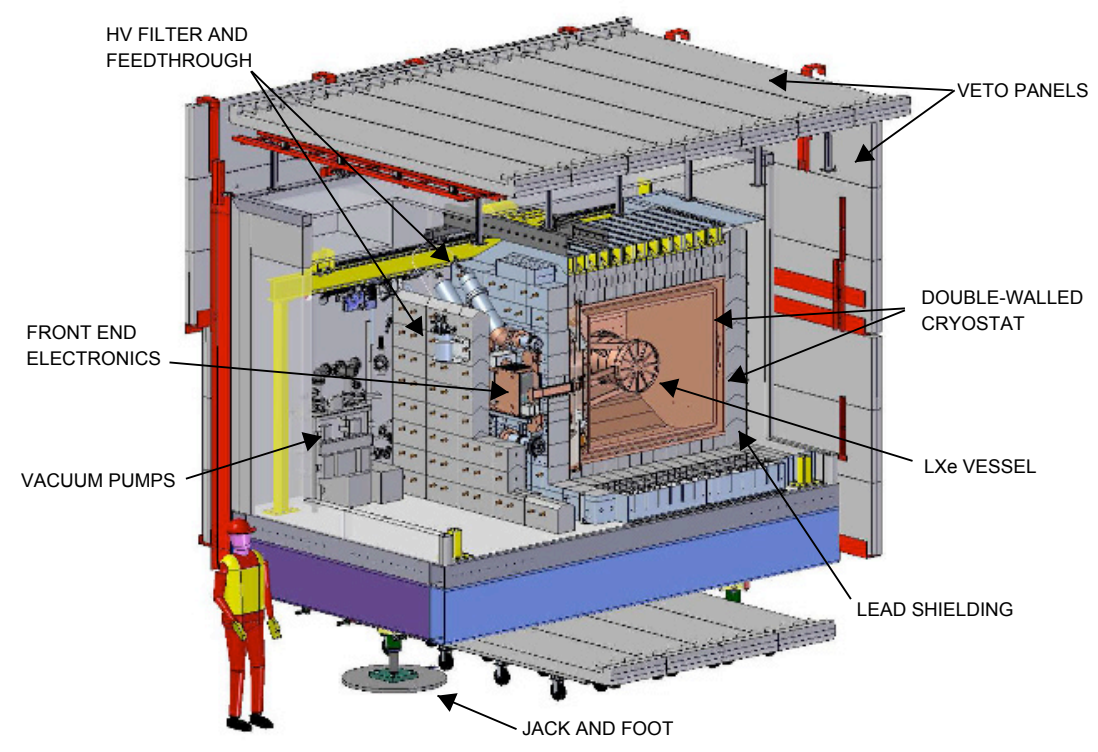

Figure 3. A drawing of the EXO-200 detector and shielding setup at WIPP.

\section{EXO-200 Results}

In 2011, the EXO collaboration published the first results from EXO-200, measuring $2 v \beta \beta$ in ${ }^{136} \mathrm{Xe}$ for the first time [4]. With just 31 live-days of data, the decay was measured with a half-life of (2.11 \pm 0.04 stat \pm 0.21 sys $) \times 10^{21}$ years. This is the longest directly measured half-life of any radioisotope.

In 2012, the EXO collaboration published the first results for their $0 v \beta \beta$ search [5]. With 120.7 days livetime and a $98.5 \mathrm{~kg}$ fiducial volume, a maximum likelihood fit was performed simultaneously on the SS and MS spectra. The fitted spectra are shown in Figure 5, with the right plot zoomed into the region of interest. In the $1 \sigma(2 \sigma)$ region of interest (ROI), 1 (5) events were observed, with a fitted expected background of $4.1 \pm 0.3(7.5 \pm 0.5)$ events. This corresponds to a $90 \%$ C.L. limit of $T_{1 / 2}^{0 \nu \beta \beta}>1.6 \times 10^{25}$ years.

The fitted background corresponds to $\sim 60$ counts $/ \pm 2 \sigma \mathrm{ROI} / 140 \mathrm{~kg} / 2 \mathrm{yr}$. This is not far from the design goal of 40 counts. The limit set on the $0 v \beta \beta$ half-life corresponds to a Majorana neutrino mass of $m_{\beta \beta}<140-380 \mathrm{meV}$, depending on which nuclear matrix element is assumed.

\section{Looking Ahead}

There are two main efforts for the future of EXO. First is improvements to EXO-200 analysis. There is now considerably more data available, and an improved analysis is being developed to analyze it. The second effort is towards the next generation detector, called nEXO.

Research and development work is underway for nEXO. This includes technology efforts, such as development of barium tagging technologies, and simulation and design efforts to measure and optimize sensitivity to $0 v \beta \beta$. A diagram of a proposed nEXO design is shown in Figure 6 . The reference design is a scale-up of the EXO-200 detector, with 5 tons of enriched liquid xenon (compared to under $200 \mathrm{~kg}$ for EXO-200). The lessons learned from EXO-200 will be applied to the design. Also, 


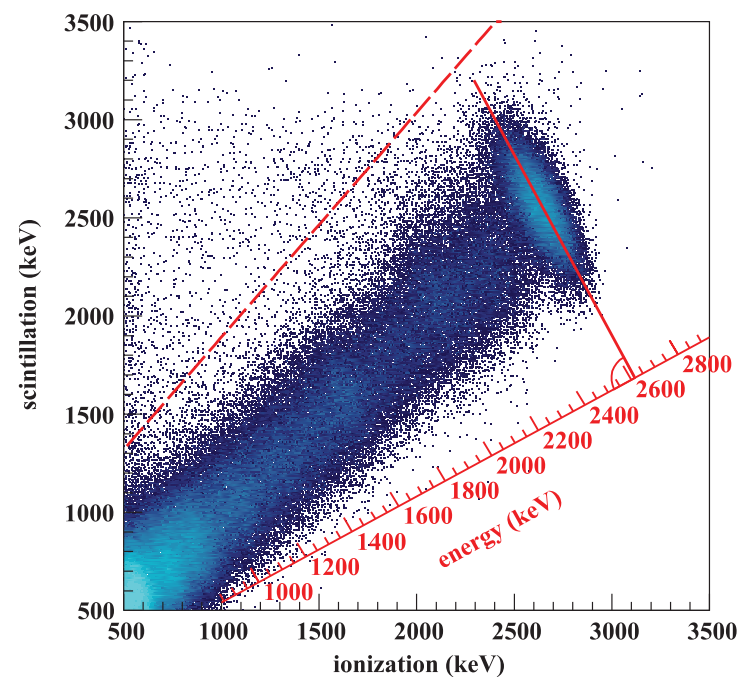

Figure 4. Plot of Thorium calibration data. The main peak at $2614 \mathrm{keV}$ from ${ }^{208} \mathrm{Tl}$ decay can be seen, and it makes the anticorrelation between scintillation and ionization clear. We choose a "rotated" energy, a linear combination of the measured scintillation and ionization energies, which gives an improved energy resolution. The ionization-only and scintillation-only energy resolutions $\left(\sigma_{E} / E\right)$ at the ${ }^{208} \mathrm{Tl}$ peak are (for SS events) $3.5 \%$ and $6.0 \%$, respectively [3], but this is improved to better than $1.8 \%$ with the "rotated" energy. The scintillation/ionization ratio can also be used to reject backgrounds such as alphas, which have a much larger scintillation fraction (the alpha events would be in the cut-off region above and to the left of this plot).

the necessary infrastructure for a barium tagging option will be included, even if barium tagging isn't ready when the data taking begins.

Figure 7 shows the projected sensitivity for EXO-200 and nEXO, as a function of the lightest neutrino mass. The present EXO-200 limit band is based on the published limit [5], and the "ultimate" sensitivity assumes radon removal (from the lead-cryostat air gap), improved analysis, and no signal in 4 years livetime. The "initial nEXO" band refers to a detector directly scaled from EXO-200, including its measured background and 10 year livetime. The "final nEXO" band refers to the same detector, but with no backgrounds other than $2 v \beta \beta$, as would be the case with barium tagging in use. The width of the horizontal bands is due to the uncertainty in nuclear matrix elements. The blue bands are $68 \% \mathrm{CL}$ intervals from oscillation experiments for the normal and inverted hierarchies. Note that nEXO, with barium tagging, should be able to completely exclude the Majorana neutrino case for the inverted hierarchy. 

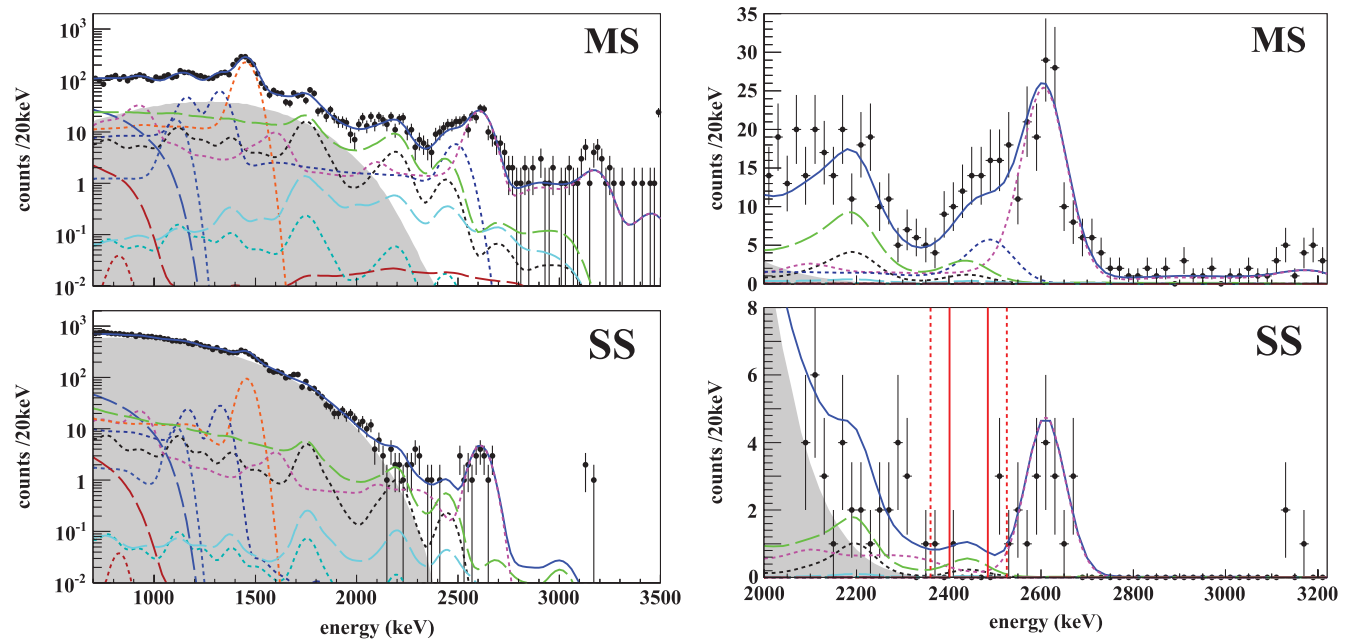

Figure 5. Fits to the low background data from 120.7 days livetime on EXO-200. The left plot shows the full fitted spectrum on a logarithmic scale, and the right plot shows the spectra zoomed in near the region of interest. The $1 \sigma(2 \sigma)$ region of interest around the Q-value are marked by vertical solid (dashed) red lines. The MS (top) and SS (bottom) spectra are fit to simultaneously. Background components are $2 v \beta \beta$ (grey region), ${ }^{40} \mathrm{~K}$ (dotted orange), ${ }^{60} \mathrm{Co}$ (dotted dark blue), ${ }^{222} \mathrm{Rn}$ in the cryostat-lead air-gap (long-dashed green), ${ }^{238} \mathrm{U}$ in the TPC vessel (dotted black), ${ }^{232} \mathrm{Th}$ in the TPC vessel (dotted magenta), ${ }^{214} \mathrm{Bi}$ on the cathode (long-dashed cyan), ${ }^{222} \mathrm{Rn}$ outside of the field cage (dotted dark cyan), ${ }^{222} \mathrm{Rn}$ in active xenon (long-dashed brown), ${ }^{135} \mathrm{Xe}$ (long-dashed blue) and ${ }^{54} \mathrm{Mn}$ (dotted brown).

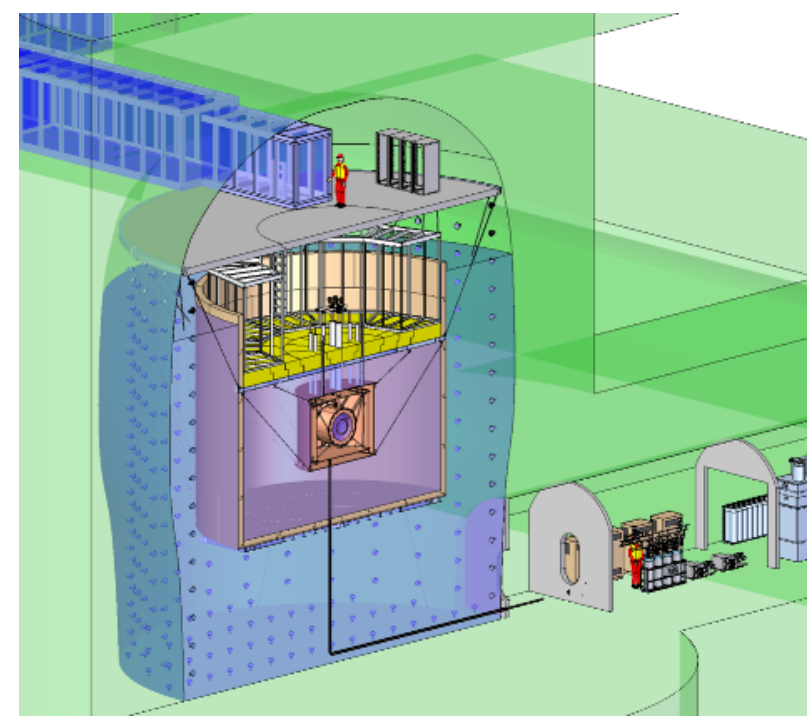

Figure 6. Drawing of a nEXO detector design. A water tank serves both as passive shielding from gamma rays, and as an active water Cherenkov muon veto. An area for barium tagging equipment is maintained above the water shield. 
INPC 2013

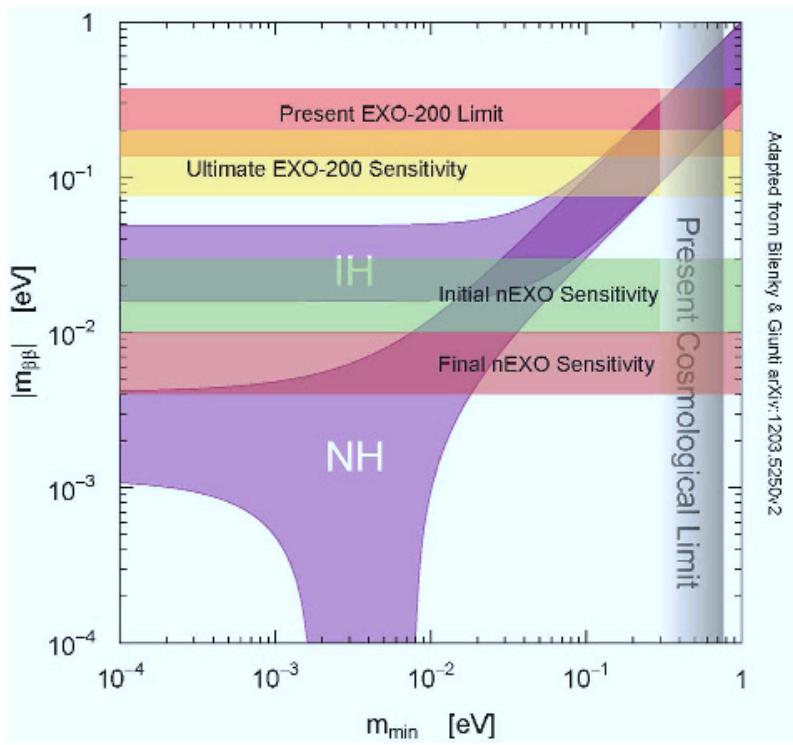

Figure 7. Sensitivities for EXO-200 and nEXO, in terms of the Majorana neutrino mass, as a function of the lightest neutrino mass.

\section{References}

[1] M. Auger, D. Auty, P. Barbeau, L. Bartoszek, E. Baussan et al., JINST 7, P05010 (2012), 1202.2192

[2] E. Conti et al. (EXO Collaboration), Phys.Rev. B68, 054201 (2003), hep-ex/0303008

[3] J. Albert et al. (EXO-200 Collaboration) (2013), 1306.6106

[4] N. Ackerman et al. (EXO-200 Collaboration), Phys.Rev.Lett. 107, 212501 (2011), 1108.4193

[5] M. Auger et al. (EXO Collaboration), Phys.Rev.Lett. 109, 032505 (2012), 1205.5608 
